\title{
GEOMETRÍA DIFERENCIAL Y TEORÍA DE LAS IDEAS: LA PRESENCIA RIEMANNIANA EN DIFERENCIA Y REPETICIÓN DE DELEUZE
}

\author{
Gonzalo Santaya* \\ doi: 10.11144/Javeriana.uph38-76.gdti
}

\section{RESUMEN}

Este artículo aporta a la teoría deleuziana de la Idea mediante un comentario a su definición técnica: "una multiplicidad definida y continua de n-dimensiones", presentada por Deleuze en el capítulo IV de Diferencia y repetición (1968). Esta definición entrelaza implícitamente la propia metafísica deleuziana de la Idea como problema virtual con una serie de nociones de la geometría diferencial desarrollada por el matemático alemán Georg B. Riemann. Con el fin de iluminar esta influencia, en un primer momento reconstruiremos los elementos fundamentales de las nociones riemannianas retomadas por Deleuze para, en un segundo momento, mostrar cómo estos elementos están supuestos por la noción deleuziana de Idea.

Palabras clave: Deleuze; Idea; virtual; Riemann; geometría diferencial

Consejo Nacional de Investigaciones Científicas y Técnicas - Universidad de Buenos Aires, Buenos Aires, Argentina.

Correo electrónico: gonsantaya@gmail.com

Para citar este artículo: Santaya, G. (2021). Geometría diferencial y teoría de las Ideas: La presencia riemanniana en Diferencia y repetición de Deleuze. Universitas Philosophica, 38(76), 49-77. ISSN 0120-5323, ISSN en línea 2346-2426. doi: 10.11144/Javeriana.uph38-76.gdti. 


\title{
DIFFERENTIAL GEOMETRY AND THEORY OF IDEAS: THE RIEMANNIAN PRESENCE IN DELEUZE'S DIFFERENCE AND REPETITION
}

\begin{abstract}
This paper contributes to clarifying Deleuze's theory of the Idea by a commentary on its technical definition: "a defined, continuous, $n$-dimensional multiplicity", presented in chapter IV of Difference and Repetition (1968). This definition implicitly intertwines Deleuze's own metaphysical view of the Idea as a virtual problem with a series of notions taken from the differential geometry developed by the German mathematician Georg B. Riemann. To clarify this influence, we will reconstruct the fundamental elements of the Riemannian notions used by Deleuze, and show how these elements are presupposed in Deleuze's concept of Idea.
\end{abstract}

Keywords: Deleuze, Idea, virtual, Riemann, differential geometry 


\section{Introducción}

La NOCión DE MUlTiplicidad o vaRiedad (Mannigfaltigkeit) de Georg Bernhard Riemann (1826-1866) es una influencia fundamental para el pensamiento deleuziano en su conjunto. Si Deleuze es el "filósofo de las multiplicidades" se deben soslayar sus referencias al "matemático de las multiplicidades". La distinción entre tipos de multiplicidad, y la indagación sobre las multiplicidades continuas o lisas que conjuntan diversas dimensiones heterogéneas, son rasgos persistentes de la obra deleuziana motivados por las ideas del matemático alemán. Este aparece en numerosas menciones, desde las monografías sobre Bergson o Foucault, hasta la caracterización de conceptos centrales como rizoma, plano de inmanencia, o Idea . En este sentido, es casi una reflexión autobiográfica la que aparece en las páginas finales de ¿Qué es la filosofía?, donde Deleuze y Guattari (1991/1993) distinguen tres tipos de interferencias que comunican los tres planos del pensamiento -el científico, el filosófico y el artístico-: "un primer tipo de interferencia surge cuando un filósofo trata de crear el concepto de una sensación, o de una función (por ejemplo, un concepto propio del espacio riemanniano, o del número irracional [...]" (Deleuze \& Guattari, 1993, p. 218; traducción modificada).

1 Deleuze definió a la filosofía como "la teoría de las multiplicidades" (Deleuze, 1995/2019, p. 51) y como una "lógica de las multiplicidades" (Deleuze, 1990/1996, pp. 233-234).

2 Riemann es citado por primera vez en Deleuze, 1966/1987a, p. 37, junto con el matemático Hermann Weyl (1922), como una influencia fundamental en la distinción bergsoniana entre dos tipos de multiplicidad. Aparece luego, como analizaremos en detalle aquí, en Deleuze, 2002, pp. 248 y 276. Contará con menciones importantes en Deleuze y Guattari, 1980/1988, pp. 39, 491 y ss., Deleuze, 1986/1987b, p. 39, Deleuze y Guattari, 1993, p. 126, entre otras. Esta insistente presencia ha solicitado la atención de diversos académicos en los últimos años, y pueden citarse numerosos trabajos que la han explorado; sin embargo, no existen -hasta donde sabemos- estudios que pretendan realizar un comentario exhaustivo a la presencia riemanniana en la teoría de la Idea deleuziana, la cual es sin duda uno de los pilares de su ontología de finales de los sesenta. Entre los principales estudios, cabe mencionar a Plotnitsky, 2006 y 2009; Duffy, 2013, pp. 162-212; Calamari, 2015; Widder, 2019. Estos últimos tres autores se centran en la influencia de Riemann en la lectura deleuziana de Bergson. Zalamea (2020) y Díez Montoya (2020) señalan la importancia de la noción en Diferencia y repetición, pero la sitúan en el contexto de un análisis más amplio y, por lo tanto, no reconstruyen de modo pormenorizado cómo el texto de Riemann se entrelaza con el de Deleuze. Jedrzejewzki, 2017, por su parte, retoma aquella aparición para reconducirla a la distinción entre "liso y estriado" en Mil mesetas. 
En este artículo pretendemos echar luz sobre la huella de esta interferencia en el concepto deleuziano de Idea, tal como este es desarrollado en el capítulo IV de Diferencia y repetición. Allí, siguiendo el "empleo riemanniano de la palabra 'multiplicidad', Deleuze redefine esta noción tan cara a la historia de la metafísica occidental como "una multiplicidad definida y continua de $n$ dimensiones" (Deleuze, 1968/2002, pp. 276-277).

Unas páginas antes de presentar esta definición, el célebre capítulo III de $D i$ ferencia y repetición ("La imagen del pensamiento") anunciaba ya la importancia de la geometría riemanniana para el proyecto filosófico de Deleuze -el de un empirismo trascendental fundado en una teoría de las Ideas entendidas como problemas-. Allí leemos:

Lo esencial es que, en el seno de los problemas, se hace una génesis de la verdad, una producción de lo verdadero en el pensamiento [...]. Para eso, es suficiente con renunciar a copiar los problemas sobre las proposiciones posibles, y con renunciar a definir la verdad de los problemas por la posibilidad de recibir una resolución. [...] Sin esa inversión, la famosa revolución copernicana no es nada. Por eso no hay revolución mientras uno se quede en la geometría de Euclides: es preciso llegar hasta una geometría de la razón suficiente, geometría diferencial de tipo riemanniano, que tiende a engendrar lo discontinuo a partir de lo continuo, o a fundar las soluciones en las condiciones de los problemas (Deleuze, 2002, pp. 247-248).

En el campo de la geometría, Riemann viene a romper con la visión euclidiana, todavía muy ligada a la "ilusión" que supone que los teoremas son verdades preexistentes a su generación, descubiertos por deducción a partir de axiomas y definiciones puestos como primeras verdades autoevidentes, para "justificar" la validez a priori de la forma del espacio adaptada a nuestra representación ${ }^{3}$. La geometría diferencial, en cambio, funda las soluciones en las condiciones del problema, o -lo que parece ser lo mismo, según el fragmento citado-engendra lo discontinuo a partir de lo continuo. Deleuze adelanta aquí una cierta comprensión de los términos

3 Las "ilusiones" que afectan al pensamiento -tanto "natural" como "filosófico" - implican tomar como punto de partida aquello que es ya de por sí la solución a un problema, impidiendo acceder a esa dimensión virtual o problemática que funda las soluciones (Deleuze las define en 2002, pp. 239-241 y pp. 244-246). 
"problema-solución" -en línea con los de "continuidad-discontinuidad"- en un trasfondo ontológico -en línea con el principio de razón suficiente-. Dicho trasfondo será ulteriormente desarrollado en los capítulos IV y V de Diferencia y repetición a través de los conceptos de Idea e intensidad, mediante los cuales Deleuze pretende fundar una filosofía de la diferencia que subvierta el lugar de la representación en el pensamiento, imperante a lo largo de la tradición occidental.

Concretamente, el capítulo IV se centra en el concepto de problema identificado con la Idea-, y lo desarrolla en resonancia con herramientas matemáticas: el cálculo diferencial, los métodos de resolución de ecuaciones algebraicas de Abel y Galois, y las filosofías de Lautman (2011) y Vuillemin (1962). De la "interferencia" con estas fuentes, Deleuze extrae herramientas expresivas para caracterizar la Idea-problema como principio de razón suficiente para una filosofía diferencial: un continuum virtual, a la vez des-fondamiento e instancia genética de la experiencia actual, compuesto por elementos diferenciales indeterminados, los cuales, existiendo solo en el seno de relaciones de determinación recíproca, engendran singularidades que determinan completamente las condiciones del problema (autodeterminación inmanente), estructurando la producción de una solución ${ }^{4}$. La multiplicidad de Riemann interfiere en el curso de esta exposición ontológica para caracterizar a la Idea como estructura diferencial $n$-dimensional que conjunta diferentes posibilidades de construcción espaciotemporales como casos de solución -tal como el espacio euclidiano es un caso posible de la multiplicidad riemanniana-.

Cabe aclarar que esta "ruptura con Euclides" sugerida por la geometría diferencial no implica una negación de la relevancia de la geometría euclidiana, sino una ruptura con la concepción que la hipostasiaba acríticamente como la única geometría posible y adecuada al espacio físico. En una entrevista de 1968, Deleuze (2002/2005) recalca este punto, comparando la historia de la matemática con la de la filosofía: "la filosofía no ha hecho revoluciones o investigaciones comparables a las que se han llevado a cabo en las ciencias [...]. Platón, Kant, etcétera, siguen siendo fundamentales, sin duda. Pero las geometrías no euclidianas no

4 Hemos desarrollado el detalle de este proceso ontológico de determinación fundado en el cálculo diferencial en Santaya, 2015 y 2017. 
impiden que Euclides siga siendo fundamental para la geometría” (p. 183). Así como no se trata de "anular" el kantismo, o el platonismo, por medio de una "inversión” de los mismos (a la que Deleuze nos convoca en la construcción de su ontología), las geometrías no euclidianas no surgieron como un intento de anular la geometría euclidiana, sino como un proceso de libre experimentación a partir de ella, revelando para la geometría nuevas direcciones y campos de análisis. Realizar en filosofía una revolución semejante implicará, para Deleuze, una reelaboración del concepto filosófico de Idea -en abierta discusión con Platón y Kant- afín a la reelaboración del concepto matemático de espacio que desarrolló Riemann.

En lo que sigue, atravesaremos dos momentos principales: en primer lugar (secciones 2 y 3), realizaremos una reconstrucción sintética de las nociones básicas de la geometría diferencial retomadas por Deleuze, siguiendo las fuentes en que Deleuze basa su comprensión de dicha geometría 5 . En segundo lugar (secciones 4 y 5), nos apoyaremos en esta reconstrucción para explicitar los detalles implícitos del concepto de Idea como multiplicidad, desarrollando un comentario a la definición deleuziana previamente citada.

\section{Geometría euclidiana y geometría diferencial}

Como destaca el matemático Hermann Weyl (1922), “el espacio era para los griegos el objeto de una ciencia muy clara y que no tenía para ellos ningún tipo de carácter conjetural. Sobre el modelo de esta ciencia se apoya para los antiguos el ideal de una ciencia pura: la geometría ha sido una de las manifestaciones más potentes de la soberanía del espíritu que animaba la cultura antigua" (p. 1). Desde esta visión antigua hasta el proyecto cartesiano de una mathesis universalis, "el ideal supremo fue, para toda ciencia, desarrollarse 'more

5 En efecto, no pretendemos hacer una presentación técnica y especializada de la matemática riemanniana, sino esbozar la recepción que Deleuze hizo de ella. Nos centraremos, para esto, en sus propias fuentes y referencias bibliográficas. Respecto a la obra de Riemann, seguiremos la traducción francesa de Jules Houël: "Sur les hypothèses qui servent de fondement à la géométrie", de 1898, por tratarse de la versión conocida por Deleuze (citada en Deleuze, 1987a, p. 37). Otras fuentes deleuzianas que comentan la geometría de Riemann son Weyl, 1922, Vuillemin, 1962, y Lautman, 2011; recurriremos a estas obras durante nuestro análisis. Nos apoyamos también en bibliografía adicional más actualizada sobre Riemann y su lugar en la historia de la matemática, indicada oportunamente. 
geometrico"' (p. 1) ${ }^{6}$. Esta soberanía espiritual mencionada por Weyl no puede considerarse aisladamente de un imperialismo epistemológico euclidiano. Si desde la antigüedad hasta la modernidad tardía el espíritu ha sido efectivamente el soberano, no lo fue sino en alianza con el modelo euclidiano que dictaminaba no solo la forma del espacio físico, sino de todo sistema de conocimiento científico. En este sentido, el historiador de la matemática José Babini (1977) se ha referido al predominio de Euclides como un imperio 7 . Si bien esto puede matizarse, pues los Elementos han sido también objeto de críticas y disputas que datan desde sus primeros comentadores ${ }^{8}$, lo cierto es que el corpus euclidiano sostuvo su dominio hasta comienzos del siglo XIX, y "hasta aproximadamente 1800 todos los matemáticos estaban convencidos de que la geometría euclídea era la idealización correcta de las propiedades del espacio físico y de las figuras en ese espacio" (Kline, 1992, p. 1138).

Carl Friedrich Gauss fue el primero en formular un sistema de geometría no euclidiana, pero no hizo públicos sus descubrimientos para no contrariar el "sentido común" euclidiano imperante en su momento, de la mano de la doctrina kantiana de la intuición’. Es también Gauss quien sienta las bases para la geometría riemanniana, al inventar los métodos necesarios para pensar figuras geométricas (superficies) desde una perspectiva interna, sin referirlas a un sistema de

6 «L'espace était pour les Grecs l'objet d'une science très claire et qui n'avait pour eux aucun caractère conjectural. C'est sur le modèle de cette science que s'est développée chez les anciens l'idée d'une science pure : la géométrie a été l'une des manifestations les plus puissantes du principe de la souveraineté de l'esprit qui animait la culture antique. [...] l'idéal suprême fut, pour toute science, de se développer more geometrico ». (Seguimos la versión francesa del texto de Weyl, citada por Deleuze. La traducción al español en todos los casos es propia).

7 Escribe Babini (1977): “[A] partir de entonces la matemática griega se refugia en la geometría, que pronto impondrá su imperio no solo sobre toda la matemática, sino sobre regiones vecinas. Los Elementos de Euclides [serán] la Biblia del saber matemático griego [...]. Es claro que los griegos no advirtieron que este imperialismo de la geometría, con todos sus éxitos, comportaba un sacrificio y una mutilación de la matemática" (pp.7-8).

8 Para un resumen de algunos problemas recurrentemente señalados al corpus euclidiano, véase Kline, 1992, pp. 1325-1328.

9 Tanto Kline (1992, p. 1151) como Datri (1999, p. 37) remiten al epistolario de Gauss para señalar esta reserva del matemático. Para un recorrido introductorio y sintético por la revolución de las geometrías no euclidianas, recomendamos el libro de de Datri, 1999. 
coordenadas exterior a las mismas. "La geometría sobre la superficie no depende en nada de la situación particular que la superficie ocupe en el espacio; ella consiste en relaciones que pueden ser obtenidas por medidas efectuadas sobre la superficie misma" (Weyl, 1922, p. 76) ${ }^{10}$. La superficie se vuelve en sí un espacio, no ya un objeto sumergido en el espacio. De este modo, aparece en geometría la posibilidad de considerar la curvatura como propiedad intrinseca al espacio, medible desde el espacio mismo. El grado de curvatura de un espacio incidirá en los movimientos posibles que un cuerpo o figura pueda realizar en él, y puede calcularse analizando dichos movimientos. Si la curvatura de un espacio es constante, las figuras en él podrán ser desplazadas sin deformación. A través de esta propiedad, llamada isometría (misma métrica), pueden transportarse superficies unas sobre otras, manteniendo las propiedades geométricas de una a otra. Una métrica, en sentido general, consiste en una técnica o método de determinación de magnitudes, a través de comparaciones entre ellas. Se trata de un patrón de proporcionalidad de la extensión de figuras, ángulos o líneas inmersas en un espacio ambiente común. El célebre teorema egregium gaussiano se apoya en estas nociones de métrica y curvatura para mostrar cómo una figura sobre una superficie de curvatura constante (por ejemplo, un triángulo dibujado en un cilindro) puede ser desplazada y superpuesta sobre otra parte de la superficie, sin deformación de la figura; esto no sucede si, por el contrario, queremos realizar un desplazamiento análogo en una superficie cuya curvatura no es constante -como en un elipsoide, donde la figura en cuestión puede resultar deformada tras el desplazamiento-. Estas ideas de Gauss son continuadas por Riemann, quien elaborará las condiciones matemáticas necesarias para realizar la deformación del espacio en su conjunto, pensando la matriz del espacio como una magnitud capaz de contener múltiples dimensiones heterogéneas, un complejo donde distintas relaciones métricas posibles coexisten.

10 «La géométrie sur la surface ne dépend en rien de la situation particulière que la surface occupe dans l'espace; elle consiste en relations qui peuvent être obtenues par des mesures effectuées sur la surface elle-même ». 


\section{Riemann: magnitud continua de dimensiones múltiples y la noción de métrica}

GaUss, quien conocía el genio de Riemann, solicitó que la lección inaugural de este último para obtener la habilitación como Privatdozent en Göttingen fuera sobre los fundamentos de la geometría. Así nace el texto en el que nos detendremos aquí, la influyente disertación de 1854 titulada Sobre las hipótesis que sirven de fundamento a la geometría ${ }^{11}$, donde se presenta la noción de multiplicidad o variedad. El texto no esconde las motivaciones filosóficas que lo llevan a presentar su perspectiva de una matemática preocupada por la estructura de los conceptos matemáticos que hacen posible la construcción del espacio ${ }^{12}$. Esta perspectiva es tanto más novedosa en cuanto que no hace nunca mención del postulado euclidiano sobre las paralelas - que había sido el eje fundamental para los descubridores de la geometría no euclidiana-, ni a los restantes axiomas y definiciones de Euclides, sino que su construcción del espacio descansa únicamente sobre la noción general de magnitud (Scholz, 1992, p. 28).

Riemann (1854/1898) comienza señalando que la geometría precedente se daba a sí misma, como "datos previos", el concepto de espacio y las construcciones fundamentales en él, sin dar de estas nociones sino definiciones nominales, para luego introducir algunas determinaciones esenciales bajo la forma de axiomas. Así, las relaciones mutuas entre estos datos primitivos permanecían "envueltas en misterio" y su enlace a priori, oculto. Hasta entonces, dice, no se había analizado suficientemente la noción de "magnitud de dimensiones múltiples, que

11 Como indicamos en la introducción, seguiremos la traducción francesa de Jules Houël del texto de Riemann, de 1898; las traducciones del francés al español de las citas en este artículo son propias. Una versión española a cargo de Emilio Méndez Pinto puede encontrarse en la biblioteca digital del Instituto Latinoamericano de Comunicación Educativa (Riemann, s.f.)

12 Desde una perspectiva deleuzo-guattariana, Plotnitsky (2009) presenta el trabajo de Riemann como una matemática conceptual. Vuillemin (1962) ya afirmaba el carácter axiomático del método riemanniano, donde los axiomas son una progresiva determinación analítica del concepto de "magnitud de dimensiones múltiples” (p. 407). Estas apreciaciones destacan en Riemann un procedimiento lógico diferente al de las geometrías euclidianas y no euclidianas que parten explícitamente de principios y construcciones: "de la descripción de modelos se pasa al análisis de estructuras y a la axiomática; la intuición deja lugar al concepto" [ «de la description des modèles, on passe à l'analyse des structures et à l'axiomatique ; l'intuition fait place au concept $»]$. (p. 408). 
comprende como caso particular las magnitudes extensas" (pp. 280-281) ${ }^{13}$. La concepción euclidiana -e incluso la no euclidiana- era aún demasiado empiri$c a$, sus principios fundamentales y construcciones básicas permanecían en cierto modo exteriores unos a otras, no se veía la necesidad interna que los conectaba. La indagación de Riemann se dirigió entonces al concepto de magnitud como fundamento de esa necesidad.

La "magnitud de dimensiones múltiples" es lo que Riemann llamará multiplicidad o variedad. El concepto de magnitud, señala, "solo es posible allí donde existe un concepto general que permita diferentes modos de determinación. En tanto sea posible, o no, pasar de uno a otro de estos modos de determinación de manera continua, ellos forman una variedad continua o una variedad discreta" (Riemann, 1898, p. 282) ${ }^{14}$. La distinción entre discreto y continuo implica una diferencia de naturaleza fundamental. De esta distinción, son las multiplicidades continuas las que captan toda la atención de Riemann (recordemos el pasaje de Deleuze citado previamente, que señalaba la importancia de engendrar lo discontinuo a partir de lo continuo para no caer en la ilusión que calcaba los problemas a partir de las soluciones). Son continuas aquellas multiplicidades que permiten operar un pasaje continuo entre los distintos modos de determinación, mientras que las discretas serán aquellas cuyos modos de determinación no admiten esta "fusión" entre sus elementos ${ }^{15}$. Las multiplicidades discretas se definen por el número de sus elementos, y presuponen la unidad como determinación fundamental a priori para la numeración. El conjunto de monedas en mi bolsillo, por ejemplo, forma una

13 «grandeurs de dimensions multiples, contenant comme case particulier les grandeurs étendues ».

14 « Les concepts de grandeur ne sont possibles que là où il existe un concept général qui permette différents modes de détermination. Suivant qu'il est, ou non, possible de passer de l'un de modes de détermination à un autre, d'une manière continue, ils forment une variété continue ou une variété discrète ».

15 Se ha afirmado que Riemann piensa este modo de transición continua inspirado en el trabajo del psicólogo Johann Friedrich Herbart, particularmente en su noción de "formas seriales" (Reihenformen): una experimentación mental que recorre una serie de representaciones individuales de manera tal que, sin destruir sus propiedades singulares, las fusiona unas a otras en una transición continua o gradual (Scholz, 1992, p. 22). Sin embargo, a la hora de pensar la continuidad desde un punto de vista estrictamente matemático, Riemann seguirá -de acuerdo con Vuillemin (1962, p. 407) - un criterio estático o estructural (véase abajo, nota 23). Para un análisis más detallado de las posibles influencias psicologistas de Riemann, véase Boi, 1995, pp. 132-134. 
multiplicidad numerable, discreta y finita: son cinco. En cambio, el sistema de los lugares que estas monedas pueden ocupar forma una multiplicidad continua y no numerable, ya que en sus modificaciones recíprocas estos espacios pueden trasladarse unos sobre otros mediante desplazamientos continuos. Riemann llama puntos a los componentes de una multiplicidad continua y elementos a los componentes de una multiplicidad discreta ${ }^{16}$. Son pocos los ejemplos de multiplicidades continuas relativas a objetos de la experiencia, donde predominan las multiplicidades discretas - la mayoría de los conceptos empíricos son una generalidad que abarca o subsume casos particulares, y se determinan como magnitud por el número de casos abarcados-. Riemann menciona solo dos ejemplos de multiplicidades continuas "empíricas": los lugares y los colores. Pero en las altas matemáticas, afirma, ellas aparecen por todas partes (Riemann, 1898, p. 282).

Una magnitud continua se considera de dimensiones múltiples cuando la determinación de cada uno de sus puntos remite a una serie de magnitudes diferentes: tantas como dimensiones posea dicha magnitud. Ellas son los "modos de determinación”. Por ejemplo, un punto en los ejes cartesianos tradicionales es una magnitud de dos dimensiones, pues su localización se determina en función de dos coordenadas $(x, y)$. Pero esta noción general no se reduce solamente al espacio geométrico. Hermann Weyl da algunos ejemplos para pensar la cuestión de la dimensionalidad y de distintos tipos de magnitudes que pueden construirse a partir de ella:

Los estados de equilibrio de un gas perfecto se diferencian unos de otros por las variables independientes presión y temperatura, ellos forman entonces una multiplicidad de dos dimensiones, como los puntos sobre una esfera, o los sonidos simples, que se definen por la intensidad y la cualidad. Conforme a la teoría psicológica, los colores forman una multiplicidad de tres dimensiones porque, siguiendo esta teoría, la percepción del color está determinada sobre la retina por la combinación de tres procedimientos químicos: el negro-blanco, el rojo-verde, el amarillo-azul (Weyl, 1922, p. 72) ${ }^{17}$.

16 La concepción de multiplicidad discreta puede leerse como una caracterización avant la lettre de la célebre noción cantoriana de "conjunto" (Mengue). Sobre los usos de estos términos en la historia de la matemática ver Torretti, 1998, pp. 7-8.

17 «Les états d'équilibre d'un gaz parfait se différencient les uns des autres par les variables indépendantes pression et température, ils forment donc une multiplicité à deux dimensions, comme les points 
Las multiplicidades riemannianas, entonces, pueden remitir a tipos de espacios que se alejan de las representaciones geométricas tradicionales: espacios fisicos de presión y temperatura, espacios cromáticos, etc. Las dimensiones que definen dichos espacios no son necesariamente homogéneas entre sí, en tanto la comparación directa de un segmento dado de una dimensión con uno de otra puede no ser posible en principio. "Ante una tal multiplicidad", escribe Vuillemin (1962), "puede que no dispongamos de instrumento de medida"; y continúa en nota a pie:

podemos [...] comparar la distancia vertical entre dos puntos con la distancia horizontal entre otros dos, porque podemos aplicar una medida primero al primer par y luego al segundo. Pero no podemos comparar la diferencia entre dos sonidos de igual altura e intensidad diferente con dos sonidos de intensidad igual y altura diferente" (p. 409) ${ }^{18}$.

Las dimensiones de la multiplicidad sonora, a diferencia de las del espacio euclidiano, son heterogéneas entre sí y no admiten la comparación directa entre sus partes sin la construcción de funciones que establezcan un criterio de traducción entre ellas (lo que añadirá complejidad a la métrica riemanniana). Para otras multiplicidades, esas dimensiones pueden ser muy numerosas - por derecho, infinitas-. En este sentido, se superan las geometrías de Gauss, Lobachevsky y Boylai, que se mantenían estrictamente en $\mathrm{R}^{3}$ (es decir, el espacio euclidiano tridimensional, definido por el cruce ortogonal de tres rectas reales). Riemann muestra cómo es posible construir una magnitud $n$-dimensional mediante la sucesiva adición de dimensiones $(n+1)$, y recíprocamente, cómo descomponer una magnitud $n$-dimensional mediante una sucesiva sustracción $(n-1)$. En el

sur une sphère, ou les sons simples qui se définissent par l'intensité et la qualité. Conformément à la théorie physiologique, les couleurs forment une multiplicité à trois dimensions, car d'après cette théorie la perception de la couleur est déterminée sur la rétine par la combinaison de trois procédés chimiques: le noir-blanc, le rouge-vert, le jaune-bleu ».

18 « Devant une telle multiplicité, il se peut que nous ne disposions pas d'instrument de mesure; [...] nous pouvons [...] comparer la distance verticale de deux points avec la distance horizontale de deux autres, parce que nous pouvons appliquer une mesure d'abord à la première paire et ensuite à la seconde. Mais nous ne pouvons pas comparer la différence entre deux sons de hauteur égale et d'intensité différente avec deux sons d'intensité égale et hauteur différente ». Deleuze y Guattari (1988, p. 491), retomarán estas referencias de Vuillemin a la hora de definir su concepto de "espacio liso", siguiendo los lineamientos de Riemann. 
primer caso, partimos del modo más simple de determinación continua cuando, dado un punto, no podemos desplazarnos sino en un solo sentido y con dos direcciones -por ejemplo, hacia adelante y hacia atrás-, lo cual nos da la primera dimensión. Si luego podemos combinar todos los puntos de este desplazamiento con uno hacia arriba y hacia abajo, habremos construido una 2-multiplicidad o multiplicidad de dos dimensiones: el plano euclidiano $\left(R^{2}\right)$. En una definición por recursividad, este procedimiento puede repetirse indefinidamente posibilitando variedades $n$-dimensionales, no intuibles como espacios para dimensiones mayores a 3, pero conceptualmente posibles. A la inversa, es posible reducir el número de dimensiones en una multiplicidad $n$-dimensional mediante progresivas reducciones $(n-1)$, manteniendo "fija" una de las direcciones posibles de variación, y variando el fenómeno en todas las restantes.

Luego de presentar este concepto, Riemann se ocupa del problema de su métrica, mediante la cual se determina la cuestión de las posibilidades de construcción de figuras y sus modos de conexión y pasaje entre diferentes regiones de la multiplicidad, estableciendo transportes o transformaciones en sus coordenadas. En el caso de las multiplicidades discretas, su métrica viene dada de antemano por la posibilidad de la numeración de sus elementos. Pero en multiplicidades continuas esta métrica es algo que debe asignarse: cuál sea la unidad de base y cómo se traslada por las distintas regiones de la multiplicidad es un problema abierto. El espacio euclidiano es un caso particular de una magnitud continua de tres dimensiones provista de una métrica constante: una unidad extensa fija arbitrariamente seleccionada (como un segmento de un centímetro, un metro o un ángulo recto) se desplaza de arriba a abajo, de derecha a izquierda, de adelante a atrás, a través de todas las regiones de ese espacio, sin que las propiedades de esa unidad se modifiquen; también es posible agrandar o achicar arbitrariamente un círculo o un cuadrado sin modificar sus propiedades internas. Siguiendo el teorema egregium de Gauss, estas modificaciones pueden realizarse incluso entre ciertos espacios curvos, cuando la curvatura es constante. El cálculo de una métrica y la determinación de sus propiedades puede realizarse -tal como había mostrado Gauss- mediante un análisis local o intrínseco a la multiplicidad. Para un observador situado sobre un punto de una superficie de dos dimensiones, el estudio de su geometría solo puede realizarse desde ella, partiendo del entorno inmediato o vecindad de sus puntos circundantes más próximos. La técnica consiste allí en 
determinar ecuaciones que establezcan los vectores ortogonales sobre el plano tangente a la superficie en el punto, y a partir de allí explorar las posibilidades de traslación y conexión con otros puntos, rodeados a su vez por otros entornos o vecindades y provistos de sus propios planos tangentes.

La asignación de una métrica a una $n$-multiplicidad riemanniana consiste en una generalización de este método, que se vuelve tanto más compleja en cuanto puede poseer una curvatura variable a través de sus $n$ dimensiones heterogéneas, entrelazadas de diferentes modos en cada punto. Riemann supondrá que la vecindad infinitesimal de un punto dado puede equipararse a su plano tangente, de modo tal que localmente, en sus "partes" infinitesimales, las coordenadas de la multiplicidad definan un espacio de tipo euclidiano ("plano"), aunque la exploración de sus conexiones puede revelar globalmente una estructura no euclidiana. La conexión entre las partes se realiza mediante la asignación de funciones integrales que construyen progresivamente el espacio. Este procedimiento corresponde a la asignación de una métrica, esencial para la determinación global de la multiplicidad. Como afirma Luciano Boi (1995): “a cada elección de la función que determina la métrica corresponde un espacio diferente que caracteriza la variedad. Así, el concepto de espacio se encuentra no solamente aclarado, sino especificado y relativizado por el de métrica" (p. 164). Esto convierte la multiplicidad en un campo de experimentación, una matriz de coexistencia de diversos espacios posibles. A continuación, veremos cómo Deleuze rescata estos rasgos y los redespliega en su teoría de la Idea.

4. Riemann en Deleuze: dimensionalidad, continuidad, definición

Como adelantamos, el capítulo IV de Diferencia y repetición presenta la definición técnica de la Idea como multiplicidad, donde entra en juego el aparato conceptual riemanniano:

Una Idea es una multiplicidad definida y continua, de $n$ dimensiones. El color, o más bien la Idea de color, es una multiplicidad de tres dimensiones. Por dimensiones es preciso entender las variables o coordenadas de las que depende un fenómeno; por continuidad es preciso entender el conjunto de las relaciones entre los cambios de esas variables, por ejemplo, una forma cuadrática de los diferenciales de las coordenadas; por definición, es necesario 
entender los elementos recíprocamente determinados por estas relaciones, que no pueden cambiar sin que la multiplicidad cambie de orden y de métrica (Deleuze, 2002, pp. 277-278).

La exposición de la Idea como multiplicidad la enfoca entonces desde el punto de vista de su referencia a un fenómeno actual que, de algún modo, depende de ella. Todo fenómeno actual remite a una multiplicidad en la medida en que encarna una Idea virtual, compuesta por: (a) una cierta cantidad de dimensiones; (b) un conjunto de relaciones entre las variaciones en esas dimensiones, que conforma un continuum; y (c) una serie de elementos recíprocamente determinados por esas relaciones, resultando en un orden y una métrica. Nos dedicamos a continuación a un comentario pormenorizado de este pasaje denso en tecnicismos, articulándolo con las nociones previamente señaladas, para explicitar lo implícito.

\section{a) DimenSIONALIDAD}

Una Idea posee una determinada cantidad de dimensiones, entendiendo por ellas las "variables o coordenadas de las que depende un fenómeno". Si bien todo fenómeno implica un cierto tipo de espacio y una duración en los que se despliega, estos no necesariamente obedecerán a los sistemas de coordenadas euclidianos o cartesianos. La Idea deleuziana le apuesta a pensar filosóficamente la génesis de espacios diversos, más allá del receptáculo tridimensional "vacío" de la representación clásica ${ }^{19}$. El fenómeno será entonces pensado según cómo se constituye el espacio que ocupa, sin suponerlo previamente como el recipiente donde el fenómeno aparece. En este sentido, la manifestación de un fenómeno es coetánea a la de un cierto número de coordenadas, de las cuales depende.

La multiplicidad "color" es un espacio cromático de tres dimensiones que abarca la totalidad de las variaciones cualitativas de los colores empíricos. La Idea de color, multiplicidad tridimensional referida a una diversidad de fenómenos, puede pensarse desde el "movimiento cromático" de un punto gris atravesando diferentes espectros, siguiendo las variables o coordenadas negro-blanco, rojoverde, amarillo-azul -como indicaba Weyl-. Las dimensiones aparecen como

19 Deleuze (2002) se refiere a espacios de tipo “geométrico, físico, biopsíquico, social y lingüístico” (p. 93).. 
consecuencia de la libre variación continua, según las direcciones sobre las cuales el punto podía variar. En la multiplicidad riemanniana asistíamos a una coordinación entre diferentes coordenadas heterogéneas que habilitaban la determinación de puntos con sus entornos o vecindades, pero donde los modos de conexión y desplazamiento posibles entre ellos debían ser construidos. De este modo, cada color actual, cualitativamente distinguible en la experiencia, sería idealmente definido como un punto, rodeado por un entorno o una vecindad dentro de la 3-variedad "color", remitiendo a un sistema de coordenadas que permite codificar su interconexión con otras vecindades, siguiendo tres direcciones. Puesto que en la transición entre dos colores ninguna es "la menor posible", la conexión supone un pasaje continuo. De ahí que las relaciones entre las coordenadas remitan a un concepto de continuidad, que conforma el segundo elemento del concepto de Idea.

\section{b) CONTINUIDAD}

Si bien la apelación a esta variación cromática nos sirve como ejemplo de una variación continua, lo cierto es que ella permanece atada a la intuición sensible, insuficiente para pensar la multiplicidad como un ámbito trascendental. El campo trascendental deleuziano está caracterizado por una paradójica noción de la variación continua. Al presentar los primeros lineamientos del concepto de Idea, de la mano del cálculo diferencial, Deleuze (2002) introducía ya la noción de una variación continua en la que impugnaba el recurso a lo empírico:

Pero si es verdad que lo continuo debe relacionarse con la Idea y con su uso problemático, lo es con la condición de no definirse ya por caracteres tomados de la intuición sensible, o hasta geométrica [...]. Lo continuo no pertenece verdaderamente a la Idea, sino en la medida en que se determina una causa ideal de la continuidad (p. 261; énfasis añadido).

Se trata, según esa presentación, de alcanzar un fondo continuo que disuelva toda forma de identidad tanto general como particular (que eran los caracteres propios de las multiplicidades discretas). Los elementos diferenciales escapan a toda forma de intuición; sin embargo, determinan cambios en estas formas, en tanto el cálculo diferencial determina un cambio de función (de primitiva a 
derivada) allí donde opera ${ }^{20}$. Como vimos, la multiplicidad de Riemann se define a su vez por elementos diferenciales, en tanto las funciones que determinan sus partes espaciales suponen la conexión de entornos infinitesimales.

Nuestro pasaje define la continuidad por "el conjunto de relaciones entre los cambios de las variables" que determinan un fenómeno (y no por la variación fenoménica misma). El ejemplo de Deleuze en este punto es tomado a la letra de la variedad descrita por Riemann en su disertación. La "forma cuadrática de los diferenciales de las coordenadas" es la función que el matemático elije para definir el elemento lineal de la multiplicidad: $d s^{2}=\sum(d x)^{2}$. Esto significa que, dado un punto de la multiplicidad, sus distancias con otros puntos de su entorno infinitesimal se comportan de manera euclidiana ${ }^{21}$, definidas por una sumatoria $(\Sigma)$ de diferencias infinitamente pequeñas $(d x)$, tantas como dimensiones tenga la multiplicidad. Riemann (1898) se ciñe explícitamente a esta expresión por ser "la más simple" y porque de otro modo "sus resultados no podrían ser expresados geométricamente” (p. 287) 22 . Esta elección de la función lineal determina la forma de las coordenadas en la vecindad de un punto P. La continuidad es un requisito impuesto a la multiplicidad riemanniana que garantiza una variación suave de punto a punto en la construcción progresiva de las líneas que unen diferentes vecindades de la multiplicidad. Al igual que en el cálculo diferencial tradicional, se busca la posibilidad de un cálculo de derivadas en todas las funciones que determinan los puntos del espacio ${ }^{23}$.

20 Hemos indagado en la naturaleza de esa continuidad a partir de las fuentes deleuzianas en Santaya, 2017.

21 La fórmula de la distancia entre puntos en el plano euclidiano-cartesiano 2-dimensional se calcula (considerándola como la hipotenusa de un triángulo rectángulo y siguiendo la regla del teorema de Pitágoras) mediante la raíz cuadrada de la suma de los cuadrados de las diferencias entre los valores que lo sitúan en las coordenadas. Formalmente, para un punto $P_{1}=\left(x_{1}, y_{1}\right)$ y un punto $P_{2}=\left(x_{2}, y_{2}\right)$, la distancia entre ellos se calcula mediante la expresión $\sqrt{\left(x_{2}-x_{1}\right)^{2}+\left(y_{2}-y_{1}\right)^{2}}$. Si consideramos las diferencias sumadas como infinitesimales $(d x)$, esta fórmula puede trasladarse al estudio de los puntos en una 2-superficie (como Gauss en su enfoque intrínseco), o bien, como hace Riemann aquí, a espacios $n$-dimensionales.

22 «es résultats ne pourraient s'exprimer géométriquement ».

23 Vuillemin (1962) rescata entre las obras de Riemann un "fragmento filosófico" en el que este explica la noción newtoniana de "pasaje al límite" como un pasaje "por un número finito de grados 
La continuidad ya no es desde entonces una mera variación intuitiva "sin saltos", sino un criterio de conexión entre regímenes heterogéneos (diferentes funciones). Al comienzo del capítulo IV de Diferencia y repetición, esta definición de la continuidad en la Idea era descrita a través del concepto de corte o límite como aquello que posibilita la continuidad, en tanto causa ideal de la misma. El par continuidad/corte marca el índice de ese cambio de función supuesto por la causa ideal de la continuidad. Desde un punto de vista metafísico, los elementos diferenciales conllevan una suerte de continuidad virtual, en la medida en que determinan conexiones entre regímenes de variación o funciones heterogéneas actuales -una continuidad superior que articula diferentes regímenes de variación continua, como por ejemplo diferentes curvas matemáticas (Deleuze, 2002, pp. 263-264; Santaya, 2017)-.

De este modo se caracteriza lo diferencial como el elemento básico de la Idea, cuyas relaciones marcan el pasaje a lo universal de un cierto régimen de variación individual, el cual es a la vez pasaje a una nueva variación individual: la causa ideal de la continuidad teje una cadena de enlace entre regímenes de variabilidad heterogéneos, empírica o intuitivamente continuos, mutuamente conectados o envueltos unos por otros, así como la multiplicidad continua enlaza sus modos de determinación a través de las relaciones entre los cambios de sus sistemas de coordenadas. La multiplicidad color, por ejemplo, relaciona los cambios de la variable negro-blanco, los cambios de la variable rojo-verde, y los cambios de la

intermedios, tal que las distancias entre dos grados intermedios sucesivos disminuyan todas al infinito" [ « le passage à travers un nombre fini de degrés intermédiaires, en sorte que les distances entre deux degrés intermédiaires successifs diminuent toutes à l'infini $\gg$ ] (p. 407). Vuillemin señala que Riemann se desliga aquí del carácter intuitivo o psicológico de la transición continua, para permanecer en su aspecto puramente conceptual, poniendo al cero como límite. En un artículo reciente, Widder (2019) expone la tesis de que el uso deleuziano del concepto de corte de Dedekind supera la concepción riemanniana de la multiplicidad como "plana en sus partes más pequeñas" [ "flatness in smallest parts"] (p. 331), la cual remitiría a este carácter intuitivo o empírico de la continuidad en lo infinitesimal. Widder no toma en cuenta esa caracterización de la continuidad sostenida por Riemann según el pasaje rescatado por Vuillemin. Desde el punto de vista matemático, la "planitud" de las partes infinitesimales de la multiplicidad es -al igual que la "rectitud" de los segmentos infinitesimales de una curva supuesta por el cálculo diferencial- una mera ficción que pretende simplificar un abordaje más técnico de las herramientas matemáticas. Desde un punto de vista metafísico, sin embargo, está cargado de un potencial notable, que Deleuze explota en su noción de "causa ideal de la continuidad". 
variable amarillo-azul, de modo que es posible pensar un pasaje ideal continuo que conecte los cortes entre sus variaciones. Así, el gris formado por la mezcla de rojo-verde puede comunicar con el formado por la mezcla de negro-blanco, o el verde del eje verde-rojo con el de azul-amarillo. Ese gris y ese verde marcan puntos o zonas de corte y articulación entre las coordenadas heterogéneas. Se trata, en cada caso, de situarse en una región local de la multiplicidad, determinada por ciertos entornos o vecindades, y construir desde allí las funciones que garanticen el pasaje o las conexiones con otras regiones. Para esto, hay que explorar aquellas zonas o puntos privilegiados que permiten la articulación.

Los cortes o límites, entonces, articulan las variaciones de las variables de las cuales dependen los fenómenos localizables en una multiplicidad, y, en virtud de ello, el conjunto de relaciones entre esas variables forma un continuo virtual. Vuillemin (1962), siguiendo la definición de dimensión de Poincaré (1902, pp. 45-46), señala:

Un continuo tiene $n$ dimensiones cuando puede descomponérselo en muchas partes practicando en él uno o varios cortes que sean ellos mismos continuos de $n-1$ dimensiones. El continuo de $n$ dimensiones se encuentra así definido por el continuo de $n-1$ dimensiones. Es una definición por recurrencia (p. 449) $)^{24}$.

Desde el punto de vista de las conexiones locales entre puntos, estos cortes implican la presencia de puntos singulares, los cuales marcan zonas de cambios y permiten entretejer los sistemas de coordenadas heterogéneos, como el punto gris que puede alternativamente variar hacia negro-blanco o hacia verde-rojo, o el 0 en las coordenadas cartesianas. En casos de multiplicidades más complejas, que conjuntan diversos sistemas de coordenadas heterogéneas, la localización de singularidades puede ser esencial para construir las series de funciones que constituyen el espacio. Los conceptos deleuzianos de continuidad, multiplicidad y singularidad están estrechamente vinculados. Así, Deleuze (2019) podrá decir -más de 20 años después de Diferencia y repetición-:

24 «n continu a $\mathrm{n}$ dimensions quand on peut le décomposer en plusieurs parties en y pratiquant une ou plusieurs coupures qui soient elles-mêmes des continus à $\mathrm{n}-1$ dimensions. Le continu à $\mathrm{n}$ dimensions se trouve ainsi défini par le continu à $\mathrm{n}-1$ dimensions. C'est une définition par récurrence ». 
Lo virtual nunca es independiente de las singularidades que lo recortan y lo dividen sobre el plano de inmanencia. [...] El plano se divide entonces en una multiplicidad de planos según los cortes del continuum y las divisiones del impulso que marcan una actualización de los virtuales (p. 52) 25 .

En resumen, la Idea virtual es continua en la medida en que determina mediante un conjunto de relaciones entre elementos diferenciales- un sistema de coexistencia entre diversos regímenes actuales de variación posibles, emisores de determinaciones expresables por fenómenos (espacios) diversos.

\section{c) ORDEN Y MÉTRICA}

El último punto de la definición deleuziana de la Idea remitía a los elementos diferenciales componentes de una multiplicidad a través del carácter de "definición": tras presentar la continuidad de la Idea como el conjunto de relaciones que la caracterizan, Deleuze indica que una multiplicidad es definida por "los elementos recíprocamente determinados por esas relaciones, que no pueden cambiar sin que la multiplicidad cambie de orden y de métrica”. El concepto de métrica es otra apropiación que Deleuze realiza del conjunto de nociones riemannianas, y que vincula aquí con el concepto de órdenes ideales y de elementos diferenciales correspondientes. Una Idea o multiplicidad cambia "de orden o de métrica”, siguiendo los cambios de los elementos en relación. Debemos, por lo tanto, indagar en este vínculo entre los conceptos de orden, métrica y elemento.

Con la mención al concepto de orden, Deleuze se retrotrae nuevamente a un momento previo del capítulo IV de Diferencia y repetición, donde su teoría de la Idea se asocia al trabajo de Albert Lautman. Este filósofo de las matemáticas definía las Ideas como instancias problemáticas que motivan y a la vez sobrepasan los medios de expresión científica dados por una teoría, y fuerzan a producir nuevas teorías. Las distintas teorías matemáticas son casos de solución a problemas dialécticos o ideales que esas teorías expresan, pero cuyo potencial problemático nunca agotan. Esto lleva a Lautman a redefinir la dialéctica como un movimiento

25 Sobre el plano de inmanencia como multiplicidad de planos véase también Deleuze y Guattari, 1993, p. 53. 
problemático que engendra conceptos y nociones matemáticas, sin confundirse con ellas ${ }^{26}$. La existencia de distintos tipos de objetos y teorías matemáticas permite una distinción entre órdenes ideales o dialécticos, cuya especificidad puede únicamente inferirse en el seno de dichas teorías, pues los problemas no existen aislada o independientemente de ellas. Deleuze (2002) realiza una extensión de esta dialéctica -que Lautman definía específicamente desde y para la matemática- a todos los dominios de lo real, de modo que no solo una teoría científica, sino también un fenómeno cualquiera, deberían ser considerados como casos actuales de solución a un problema dialéctico:

Los problemas son siempre dialécticos, la dialéctica no tiene otro sentido, los problemas tampoco tienen otro sentido. Lo que es matemático (o físico, o biológico, o psíquico, o sociológico...) son las soluciones. Pero es cierto, por una parte, que la naturaleza de las soluciones remite en la dialéctica misma a órdenes diferentes de problemas; $y$, por otra, que los problemas, en virtud de su inmanencia, no menos esencial que la trascendencia, se expresan ellos mismos técnicamente en ese dominio de soluciones que generan en función de su orden dialéctico. Así como la recta y el círculo encuentran su doble en la regla y el compás, cada problema dialéctico tiene su doble en un campo simbólico donde se expresa (pp. 272-273).

Este pasaje de inspiración lautmaniana ilustra el doble aspecto que la filosofía de la diferencia deleuziana atribuye a los objetos: todo objeto es doble, compuesto de una mitad virtual y una mitad actual. Deleuze presenta aquí el ejemplo de la regla y el compás como los medios de expresión en lo actual de elementos problemáticos como la recta y el círculo, los cuales ni aparecen fenoménicamente ni pueden ser efectivamente construidos por la intuición en su infinita riqueza, y sin embargo regulan la producción de medios de expresión o campos simbólicos que permiten una cierta presentación intuitiva. Dado que la experiencia y el pensamiento nos confrontan con objetos (tanto empíricos como teóricos) de diferente naturaleza, hay elementos virtuales o problemáticos de diferentes

26 Junto a Cavaillès, Lautman es uno de los introductores del formalismo alemán en Francia, lo que lo convierte en una influencia mayor para el célebre grupo Bourbaki. Sobre sus ideas acerca de la dialéctica y su relación con la matemática, puede consultarse Lautman, 2011. 
órdenes: orden matemático, o físico, o biológico, o psíquico, o sociológico, etc., diferentes dominios de expresión actual a diferentes órdenes dialécticos de problemas virtuales.

La Idea dialéctica, problemática, es un sistema de ligazones entre elementos diferenciales, un sistema de relaciones diferenciales entre elementos genéticos. Hay diferentes órdenes de Ideas, supuestos los unos por los otros, siguiendo la naturaleza ideal de las relaciones y los elementos considerados (Idea de la Idea, etc.) (Deleuze, 2002, p. 275; traducción modificada).

El "mundo de las Ideas" deleuziano es, pues, un continuum virtual hecho de elementos diferenciales; no remite lo múltiple sensible a lo Uno, ni a lo idéntico, ni a lo permanente, sino a multiplicidades problemáticas fluentes en comunicación con otras multiplicidades. Los distintos fenómenos remiten a distintos órdenes de elementos diferenciales, elementos indeterminados pero determinables únicamente en y por las relaciones que los comunican y mediante las cuales engendran las determinaciones propias de esas soluciones, sin que estas puedan agotar el potencial genético de aquellas. Fuera de estas relaciones, los elementos de la multiplicidad son en sí indeterminados: "es preciso que no tengan ni forma sensible ni significación conceptual, ni - por consiguiente- función asignable" (Deleuze, 2002, p. 278). Pero -siguiendo la caracterización de la continuidad ideal- el conjunto de sus relaciones permite establecer valores singulares que funcionan como puntos de corte y articulación entre sistemas de coordenadas heterogéneos, permitiendo construir una métrica que caracterice los modos de interconexión entre los puntos de la multiplicidad, y modulando el devenir de sus expresiones fenoménicas. Un cambio en esos elementos indeterminados conlleva un cambio de orden dialéctico, a la vez que un cambio de singularidades y de aquellas expresiones.

Desde el punto de vista de la multiplicidad riemanniana, eran las relaciones métricas las que determinaban sus fenómenos (las formas y figuras construibles en ella), mediante la asignación, ya de invariancias, ya de deformaciones, en las longitudes de segmentos lineales o en los arcos de ángulos comparados en las distintas regiones de dicha multiplicidad. Ahora bien, como vimos, la métrica podía ser constante o variable a través de las regiones de una multiplicidad. La existencia de una métrica constante supone una continuidad homogénea del espacio 
en el cual vale: un sistema de puntos conectados entre sí tal que sus distancias relativas sean mensurables de manera directa las unas con las otras, traducibles a longitudes unificadas bajo un patrón común. En caso de que la métrica sea variable -si las dimensiones de la multiplicidad son heterogéneas entre sí- ella exigirá para ser definida una construcción de funciones de traducción entre sus distintos sistemas locales de coordenadas. Una misma multiplicidad, considerada desde el punto de vista de sus relaciones diferenciales componentes, puede tolerar diferentes relaciones métricas, como diferentes instanciaciones espaciales posibles que grafican sus diferentes regiones. En este sentido, la metrización de una multiplicidad es análoga a la exploración de las posibilidades de un espacio. En el caso desarrollado por Riemann, la metrización suponía como elemento básico la forma diferencial cuadrática en las coordenadas de las vecindades de un punto $\left(d s^{2}=\Sigma(d x)^{2}\right)$, pero el concepto general de multiplicidad admite otras expresiones posibles.

Así como Deleuze operaba una extensión del concepto lautmaniano de dialéctica -llevándolo más allá de las matemáticas-, podemos decir que opera también una extensión del concepto de métrica riemanniana -llevándolo a los diferentes dominios de solución que componen la diversidad fenoménica de la experiencia y las diferentes teorías científicas-. Los distintos órdenes dialécticos (biológico, psíquico, lingüístico, etc.), como diferentes complejos estructurales supuestos los unos por los otros en el continuum virtual, remiten a distintos tipos de definición de las multiplicidades, en tanto son determinados por su instanciación en relaciones métricas actuales que desarrollan fenoménicamente un espacio asociado a la multiplicidad. Si, por un lado, esta tipología de elementos diferenciales solo puede ser inferida retroactivamente en virtud de la naturaleza de las soluciones actuales que los expresan, la topología de estas soluciones, a la inversa, no encuentra su razón suficiente sin suponer la estructura virtual de la multiplicidad subyacente. Las determinaciones métricas de las soluciones actuales permanecen como meras hipótesis empíricas si no se señala la instancia genética que las expone como un caso de solución entre otros posibles, así como el espacio euclidiano aparecía como un caso particular de la multiplicidad riemanniana. 


\section{Conclusión: la multiplicidad en el empirismo trascendental}

Si PENSAMOS EN UN CONCEPTO MÁS AMPLIO del espacio (y del tiempo) que el que la experiencia o la geometría clásica suponen, es preciso afirmar la existencia de espacios biológicos, psíquicos, lingüísticos, sociales, etc. Cada uno de estos espacios está determinado por los modos de conexión y composición de los subespacios que los componen y configuran (la exterioridad recíproca y la conexión de las partes orgánicas de un cuerpo, de las representaciones y voliciones de un sujeto psíquico, de los elementos gramaticales, sintácticos y pragmáticos de una lengua, de las instituciones y agentes de una sociedad). Todos estos espacios actuales remiten a una espacialidad virtual como su instancia genética y estructurante. En cada caso, el espacio virtual está recubierto por sus expresiones fenoménicas o empíricas, pero íntimamente entrelazada con ellas, dando lugar a lo que el empirismo trascendental deleuziano llama el "ejercicio trascendente" de las facultades:

Sea la multiplicidad lingüística, como sistema virtual de ligazones recíprocas entre "fonemas", que se encarna en las relaciones y los términos actuales de las lenguas diversas: tal multiplicidad hace posible el habla como facultad, y el objeto trascendente de ese habla [...] en el ejercicio poético del habla coextensivo a la virtualidad. Sea la multiplicidad social: determina la sociabilidad como facultad, pero también el objeto trascendente de la sociabilidad, que no puede ser vivido en las sociedades actuales donde la multiplicidad se encarna, pero que debe serlo y no puede sino serlo en el elemento de transformación de las sociedades (a saber, simplemente la libertad, siempre recubierta por los restos de un antiguo orden y las primicias de uno nuevo) (Deleuze, 2002, p. 292).

La captación de un fenómeno (lingüístico, social, biológico, psíquico...) como una actualización nos retrotrae al elemento genético de sus determinaciones. Captar un enunciado desde el ejercicio trascendente del habla conduce a la multiplicidad lingüística que posibilita el ejercicio empírico -en el cual su significación corresponde a un estado de cosas externo-. Lo que se capta en esa multiplicidad son los fonemas en sus relaciones inmanentes de ligazón múltiple, es decir, en tanto elementos diferenciales que remiten a un espacio intrínseco de 
determinación recíproca, una variedad sonora capaz de engendrar una diversidad inagotable de palabras, enunciados, lenguas, como otras tantas maneras de establecer conexiones entre palabras y cosas en el ejercicio empírico del lengua$\mathrm{je}^{27}$. Las distintas gramáticas son métricas que asocian esos elementos virtuales del ejercicio trascendente del habla, estableciendo sus reglas de conexión y dando lugar a enunciados correspondientes a lenguas actuales diversas.

Lo mismo vale para la multiplicidad social y la facultad de la sociabilidad: los roles y funciones de los individuos e instituciones de un sistema social dado establecen la métrica de una sociedad, que determina los desplazamientos y conexiones actuales para cada agente en ese sistema. Más allá de su funcionamiento habitual, esos mismos roles, funciones y agentes serán meras instanciaciones de la multiplicidad -no ya las figuras sociales que forman el cuerpo social, sino los elementos estructurales informales, subyacentes a su configuración ${ }^{28}-$. Por extraño que pueda resultar que una masa importante de agentes sociales alcance esa percepción de su espacio social como una pura variedad pasible de actualizarse de diversos modos (y por lo tanto de captarse también a sí mismos como individuaciones fortuitas), ella no deja de insistir como el fundamento ontológico de la constitución y el cambio social (el $\sin$-fondo de toda sociedad, que Deleuze llama "libertad" en la cita precedente). Los estados de excepción en la historia de las sociedades -revoluciones, guerras, luchas de las minorías, movimientos migratorios masivos, pandemias, etc.-, atestiguan la presencia de ese campo trascendental. A la inversa, toda constitución -metrización o estratificación- de un orden social es determinada por y en ese campo, que genera las condiciones de emergencia de un nuevo régimen institucional. Esto no implica una visión teleológica o determinista: tanto el momento de emergencia de la multiplicidad

27 Sobre la Idea lingüística ver Deleuze (2002, pp. 306-310). Ver también la Logica del sentido, donde Deleuze realiza el análisis de la proposición descomponiéndola en sus dimensiones, y exponiendo el sentido como cuarta dimensión (1969/2013, pp. 41-42).

28 Sobre la Idea social, véase Deleuze, 2002, pp. 281-283, 268-269, donde enlaza su teoría de la multiplicidad con los aportes estructuralistas al marxismo de Althusser, Balibar, Macherey y Rancière (1965). Por su parte, en El Anti-Edipo, Deleuze y Guattari (1972/1985, p. 234-235), recurrirán a la relación diferencial para caracterizar el plano de inmanencia propiamente capitalista, donde $d x$ representa el capital y $d y$ la fuerza de trabajo, como puras cantidades abstractas únicamente en cuya conjunción pueden emerger las instituciones y personas diversas que pueblan ese plano. 
es imprevisible desde el punto de vista de la empiria determinada por ella, como imprevisible es la nueva métrica, los nuevos regímenes pasibles de constituirse en esa emergencia.

Puesto que la Idea así caracterizada carece de forma -intuitiva o conceptual-, $y$ sin embargo determina la creación de formas, deberá plantearse al empirismo trascendental la pregunta acerca de la naturaleza de esa producción. De acuerdo con Deleuze (2002), el modo en que las multiplicidades se actualizan implica pensarlas desde las intensidades que las recorren, como fuerzas que actúan dinamizando o dramatizando la construcción de espacio-tiempos, de cualidades y extensiones, correspondientes a la estructura virtual de las Ideas: "en el orden dinámico, ya no hay ni concepto representativo, ni figura representada en un espacio preexistente. Hay una Idea y un puro dinamismo creador del espacio correspondiente" (p. 49).

Es digno de mención que Riemann, hacia el final de su disertación, señala el problema acerca de cómo construir una métrica adecuada para el análisis de los fenómenos físicos desde su noción de multiplicidad, especialmente allí donde el espacio escapa a nuestra capacidad de observación -considerando los casos de lo infinitamente grande y lo infinitamente pequeño-. Mientras que en las variedades discretas la métrica viene dada por el mismo concepto de esta variedad, en las variedades continuas ella debe ser asignada o construida. Diferentes métricas darán por resultado diferentes configuraciones fenoménicas. $\mathrm{Al}$ no poder verificar empírica o directamente qué métrica corresponde a los espacios estudiados en lo macro y lo microscópico, la inexactitud amenaza la investigación. Esto deja entonces dos caminos: "o que la realidad sobre la que se funda el espacio sea una variedad discreta, o que el fundamento de las relaciones métricas sea buscado fuera de él, en las fuerzas de ligazón que actúan en él" (Riemann, 1898, p. 297; énfasis añadido) ${ }^{29}$. Si la hipótesis (propia de las multiplicidades discretas) de la existencia de un patrón de unidad a priori para la representación del espacio queda rechazada por su insuficiencia para dar cuenta acabadamente de los fenómenos, la métrica de la multiplicidad deberá ser determinada a partir de las fuerzas físicas, de las que se debe decir entonces no solamente que habitan el espacio o la materia

29 « ou que la réalité sur laquelle est fondé l'espace forme une variété discrète, ou que le fondément des rapports métriques soit cherché en dehors de lui, dans les forces de liaison qui agissent en lui ». 
(los cuales, desde este punto de vista, comienzan a devenir indiscernibles), sino que lo estructuran, constituyen y ligan sus partes. Precisamente en este punto, que reenvía directamente al dominio de la física, Riemann finaliza su disertación. En Deleuze, el análisis de estas fuerzas de ligazón remite a la intensidad -concepto desarrollado en el capítulo V de Diferencia y repetición-, la cual, por un lado, eleva en lo empírico la emergencia de los problemas y, por otro lado, dinamiza su estructura virtual dirigiendo el proceso de actualización o resolución ${ }^{30}$. En el medio, la Idea-problema yace como el caótico espacio $n$-dimensional emisor de las singularidades expresadas por las intensidades que crean las extensiones y las métricas relativamente estables de la experiencia actual.

\section{Referencias}

Althusser, L., Balibar, É., Macherey, P., \& Rancière, J. (1965). Lire le capital. París: Maspero.

Babini, J. (1977). Newton, Leibniz y el cálculo infinitesimal. En: J. Babini (Ed.), El cálculo infinitesimal (pp. 15-39). Buenos Aires: Eudeba.

Boi, L. (1995). Le problème mathématique de l'espace. Une quête de l'intelligible. Berlín: Springer.

Calamari, M. (2015). Riemann-Weyl in Deleuze's Bergsonism and the Constitution of the Contemporary Physico-Mathematical Space. Deleuze Studies, 9(1), 59-87. https://doi.org/10.3366/dls.2015.0174

Datri, E. (1999). Geometría y realidad física de Euclides a Riemann. Buenos Aires: Eudeba.

Deleuze, G. (1987a). El bergsonismo. (Trad. L. F. Carracedo). Madrid: Cátedra. Deleuze, G. (2002). Diferencia y repetición. (Trad. H. Beccacece y M. S. Delpy). Buenos Aires: Amorrortu.

Deleuze, G. (1987b). Foucault. (Trad. J. V. Pérez). Barcelona: Paidós.

Deleuze, G. (1996). Conversaciones. (Trad. J. L. Pardo). Valencia: Pre-Textos.

30 El análisis del concepto deleuziano de intensidad excede con mucho los objetivos del presente trabajo. Para una aproximación a la noción de intensidad y sus implicancias en la filosofía deleuziana del espacio puede verse McNamara, 2018. 
Deleuze, G. (2005). La isla desierta y otros textos. Textos y entrevistas (19531974). (Trad. J. L. Pardo). Valencia : Pre-Textos.

Deleuze, G. (2013). Lógica del sentido. (Trad. M. Morey). Barcelona: Paidós.

Deleuze, G. \& Guattari, F. (1985). El Anti-Edipo. Capitalismo y esquizofrenia. (Vol. 1). (Trad. F. Monge). Barcelona: Paidós.

Deleuze, G.\&Guattari,F.(1988).Milmesetas. Capitalismoyesquizofrenia. (Vol.2). (Trad. J. V. Pérez y U. Larraceleta). Valencia: Pre-textos.

Deleuze, G. \& Guattari, F. (1993). ¿Qué es la filosofía? (Trad. T. Kauf). Barcelona: Anagrama.Deleuze, G., (2019). Lo actual y lo virtual. (Trad. R. Di Renzo). Buenos Aires: Red Editorial.

Díez Montoya, S. (2020). La ontología de los problemas de Diferencia y repetición. Universitas Philosophica, 37(74), 77-100. https://doi.org/10.11144/Javeriana.uph37-74.opdr.

Duffy, S. (2013). Deleuze and the History of Mathematics. In Defense of the New. Edimburgo: Edinburgh University Press.

Jedrzejewzki, F. (2017). Deleuze et la géométrie riemanniene: Une topologie des multiplicités. En: L. Ji, A. Papadopoulos \& S. Yamada (Eds.), From Riemann to Differential Geometry and Relativity (pp. 311-328). Cham: Springer International Publishing.

Kline, M. (1992). El pensamiento matemático de la antigüedad a nuestros dias. (Trad. M. Martínez, J. Tarrés \& A. Casal). Madrid: Alianza Editorial.

Lautman, A. (2011). Ensayos sobre la dialéctica, estructura y unidad de las matemáticas modernas (Trad. F. Zalamea). Bogotá: Universidad Nacional de Colombia.

McNamara, R. (2018). Filosofía del espacio y teoría de la acción en Gilles Deleuze. Contrastes. Revista Internacional de Filosofía, XXIII(2), 41-57. https:// doi.org/10.24310/Contrastescontrastes.v23i2.5571

Plotnitsky, A. (2006). Manifolds: On the Concept of Space in Riemann and Deleuze. En: S. Duffy (Ed.), Virtual Mathematics. The Logic of Difference (pp. 187-208). London: Clinamen Press.

Plotnitsky, A. (2009). Riemann's Conceptual Mathematics. Configurations, $17(1-2), 105-130$.

Poincaré, H. (1902). Science et hypothèse. París: Flammarion. 
Riemann, G. B. (1898). Sur les hypothèses qui servent de fondement à la géométrie (Trad. J. Houel). CEuvres mathématiques (pp. 280-299). París: Gauthier-Vilars.

Santaya, G. (2015). La Idea según Gilles Deleuze: Una aproximación desde el cálculo diferencial. Ideas. Revista de filosofía moderna y contemporánea, 1 , 134-162.

Santaya, G. (2017). El cálculo trascendental. Gilles Deleuze y el cálculo diferencial: ontología e historia. (Colección "Deleuze y las fuentes de su filosofía." Vol. IV). Buenos Aires: RAGIF Ediciones.

Scholz, E. (1992). Riemann's Vision of a New Approach to Geometry. En: L. Boi, D. Flament \& J. M. Salanskis (Eds.), 1830-1930: A Century of Geometry. Epistemology, History and Mathematics (pp. 22-34). Berlín: Springer-Verlag. Torretti, R. (1998). El paraiso de Cantor. La tradición conjuntista en la filosofia matemática. Santiago de Chile: Editorial Universitaria y Universidad Andrés Bello.

Vuillemin, J. (1962). La philosophie de l'algèbre. París: PUF.

Weyl, H. (1922). Temps, espace, matière. Leçons sur la théorie de la relativitégénérale. (Trad. G. Juvet y R. Leroy). París: Albert Blanchard.

Widder, N. (2019). The Mathematics of Continuous Multiplicities: The Role of Riemann in Deleuze's Reading of Bergson. Deleuze \& Guattari Studies, 13(3), 331-354. https://doi.org/10.3366/dlgs.2019.0361.

Zalamea, F. (2020). Diferencia y repetición: Preludios en la matemática moderna y ecos en la matemática contemporánea. Universitas Philosophica, 37(74), 139-153. https://doi.org/10.11144/Javeriana.uph37-74.drpm 